\title{
Composite follicular lymphoma and classic Hodgkin lymphoma
}

\author{
Han-Na Kim ${ }^{1}$, Min Ji Jeon ${ }^{2}$, Eun Sang $\mathrm{Yu}^{2}$, Dae Sik Kim², Chul-Won Choi ${ }^{2}$, Young Hyeh Ko ${ }^{1}$ \\ ${ }^{1}$ Department of Pathology, 2Division of Hematology-Oncology, Department of Internal Medicine, Korea University Guro Hospital, Seoul, Korea
}

Composite lymphoma is very rare and a combination of Hodgkin lymphoma and non-Hodgkin lymphoma and even histiocytic tumors can occur. Because of the unfamiliarity, not only can this cause diagnostic problems, but can also affect treatment plan. We report a case of composite lymphoma in a 40-year-old male. Initial biopsy showed a composite lymphoma of follicular lymphoma grade 1 and classic Hodgkin lymphoma. After chemotherapy, another lymph node was taken because of disease progression, which revealed follicular lymphoma, grade 3a without Hodgkin lymphoma component.

Key Words: Composite lymphoma; Follicular lymphoma; Hodgkin lymphoma

Received: September 14, 2021 Revised: September 28, 2021 Accepted: October 9, 2021

Corresponding Author: Young Hyeh Ko, MD, PhD, Department of Pathology, Korea University Guro Hospital, 148 Gurodong-ro, Guro-gu, Seoul 08308, Korea Tel: +82-2-2626-1482, Fax: +82-2-2626-1486, E-mail: yhk0310@skku.edu

Composite lymphoma was initially recognized by Custer [1] as two or more distinct lymphomas that occur in the same patient. This definition was later refined by Kim et al. [2] as simultaneous occurrence of more than one type of lymphoma in the same organ or tissue site of the same patient. Composite lympho$\mathrm{ma}$ is rare and may consist of non-Hodgkin lymphoma (NHL) with Hodgkin lymphoma (HL) [3,4], B-cell NHL with T-cell NHL [5-8], T-cell or B-cell NHL with other histologic type(s) of the same lineage [9], or NHL with histiocytic or dendritic cell tumors [10]. Excluding follicular lymphoma (FL) associated with diffuse large B-cell lymphoma, the most common composite lymphoma is $\mathrm{FL}$ of low grade associated with classic $\mathrm{HL}[3,4,11]$ followed by FL associated with mantle cell lymphoma [9,12]. Histologically, composite lymphomas display a mixed pattern, or less commonly, distinct zonal distribution of each lymphoma component $[3,5]$. Composite lymphoma may pose a diagnostic challenge, especially when two lymphoma components are mixed in the same lymph node. Herein we report a case of composite HL and FL of mixed histologic pattern.

\section{CASE REPORT}

\section{Clinical findings}

A 40-year-old man was diagnosed with HL at an outside hos- pital and was referred for treatment. He showed a neck mass and weight loss. Laboratory tests were within normal limits except for a positive hepatitis $B$ antigen test. A positron emission tomography (PET)-computed tomography (CT) scan revealed multiple right level I, II, and V hypermetabolic lymph nodes of the neck, both axillae, and retropancreatic regions. Abdominal CT scan revealed no organomegaly, but mild irregular hypermetabolism was noted in the spleen and along the marrow space by PET-CT. Under the diagnosis of classic HL, nodular sclerosis, stage IV, the patient was treated with doxorubicin, bleomycin, vinblastine, and dacarbazine (ABVD) chemotherapy, and an antiviral agent for hepatitis $B$ was administered. A few months later, abdominal CT scan revealed slightly increased sizes of multiple lymph nodes at the portocaval, aortocaval, left paraaortic, and small bowel mesentery. Lymph node biopsy from a level IV node was diagnosed as FL, grade 3a. Bone marrow biopsy was negative. Six cycles of rituximab with bendamustine were planned.

\section{Pathologic findings}

All the lymph node biopsies were reviewed. The biopsy was evaluated with immunohistochemical stains including staining for CD20, CD3, CD30, CD15, PAX5, BCL2, CD10, and Ki-67 (Leica Biosystems, Newcastle upon Tyne, UK). Immunohistochemistry was performed using a Leica BOND-III automated 
stainer (Leica Biosystems, Melbourne, Australia). Epstein-Barr virus was detected by in situ hybridization (ISH) using a Bond Ready-to-Use ISH Epstein-Barr virus (EBV)-encoded RNA probe (Leica Biosystems, Newcastle upon Tyne, UK).

The initial biopsy that was diagnosed as $\mathrm{HL}$ at outside clinic was reviewed. Enlarged follicles were found at the peripheral part of the node but the center showed diffuse area. Large neoplastic Hodgkin Reed-Sternberg cells were easily recognizable in the diffuse area but also found in the peripheral area between the neoplastic follicles. Histiocytes were mixed but eosinophils were not prominent. Reed-Sternberg cells were positive for $\mathrm{CD} 30, \mathrm{CD} 15$, and PAX5, but negative for $\mathrm{CD} 20$ and $\mathrm{CD} 3$. PAX5 staining was relatively weak in comparison with the neighboring non-neoplastic B cells. Neoplastic follicles were positive for CD20, CD10, and BCL2, but negative for CD30 and CD15. Therefore, we concluded that it was a composite lymphoma of FL grade 1 and clas- sic HL (Figs. 1A-I, 2A-C). EBV was negative in both $\mathrm{HL}$ and FL cells. The second biopsy performed in our hospital showed only FL without $\mathrm{HL}$ component but this time the grade of FL was $3 \mathrm{a}$ because of centroblastic proliferation (Fig. 2D-F).

\section{DISCUSSION}

Composite FL and $\mathrm{HL}$ is extremely rare and only 25 cases have been reported in the literature written in English to date [3,4,1116]. While composite lymphoma usually is indicated histopathologically by at least two morphologically distinct lymphomatous proliferations, the proof that these proliferations are separate and distinct neoplasms requires immunologic analysis. The true incidence of composite lymphoma may be underestimated because as in this case, separate tumor components can be easily overlooked on initial microscopic examination.
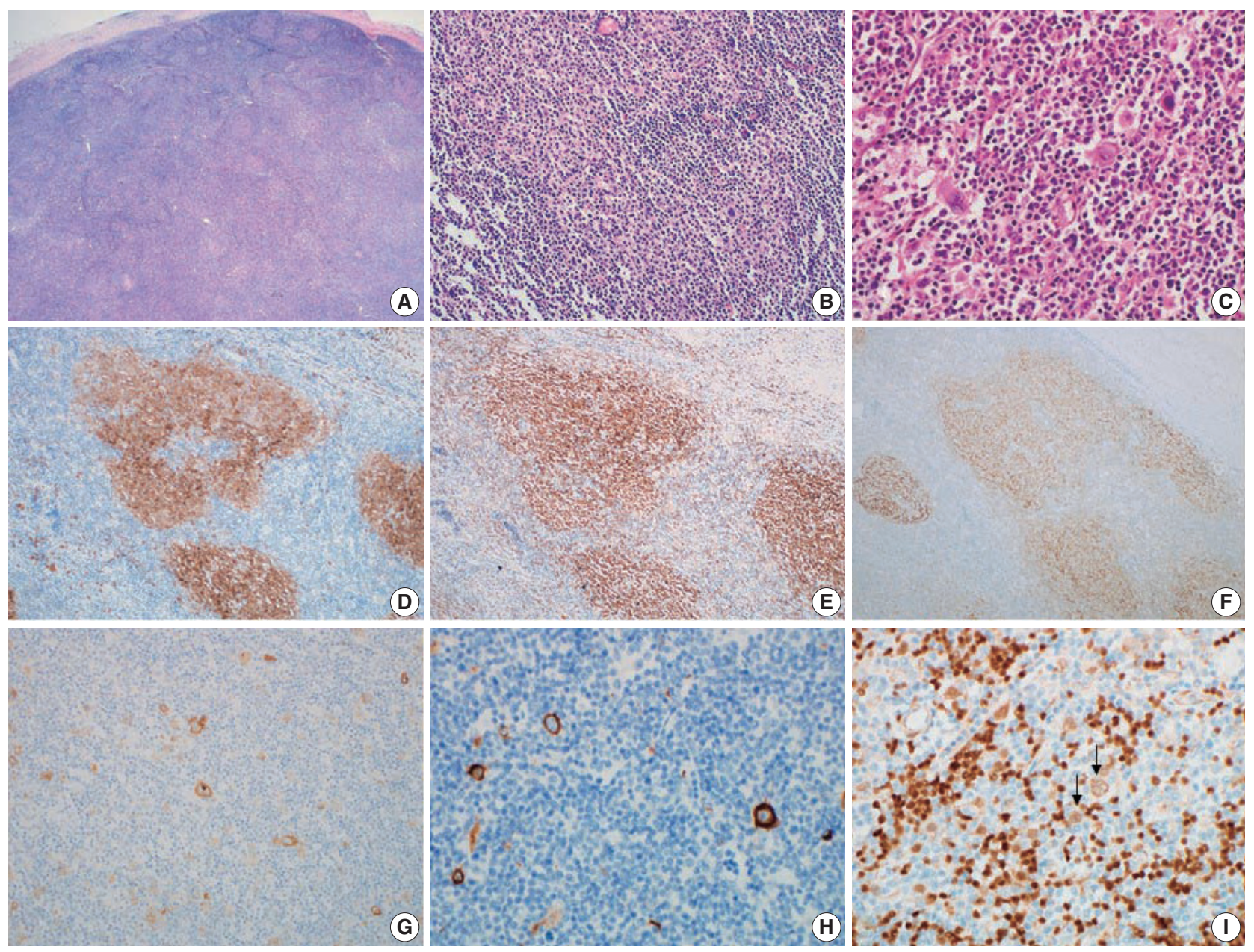

Fig. 1. Composite follicular lymphoma and Hodgkin lymphoma of initial biopsy. (A) Lymph node shows nodular area representing follicular lymphoma and diffuse area containing Hodgkin lymphoma. (B) Follicular lymphoma, grade 1. (C) Reed-Sternberg cells of Hodgkin lymphoma. (D) CD10-positive cells of follicular lymphoma. (E) BCL2-positive follicular lymphoma. (F) CD21-positve follicular dendritic cell meshwork of follicular lymphoma. (G) CD30 in Reed-Sternberg cells. (H) CD15 in Reed-Sternberg cells. (I) PAX5 weak positive in Reed-Sternberg cells (arrows). 

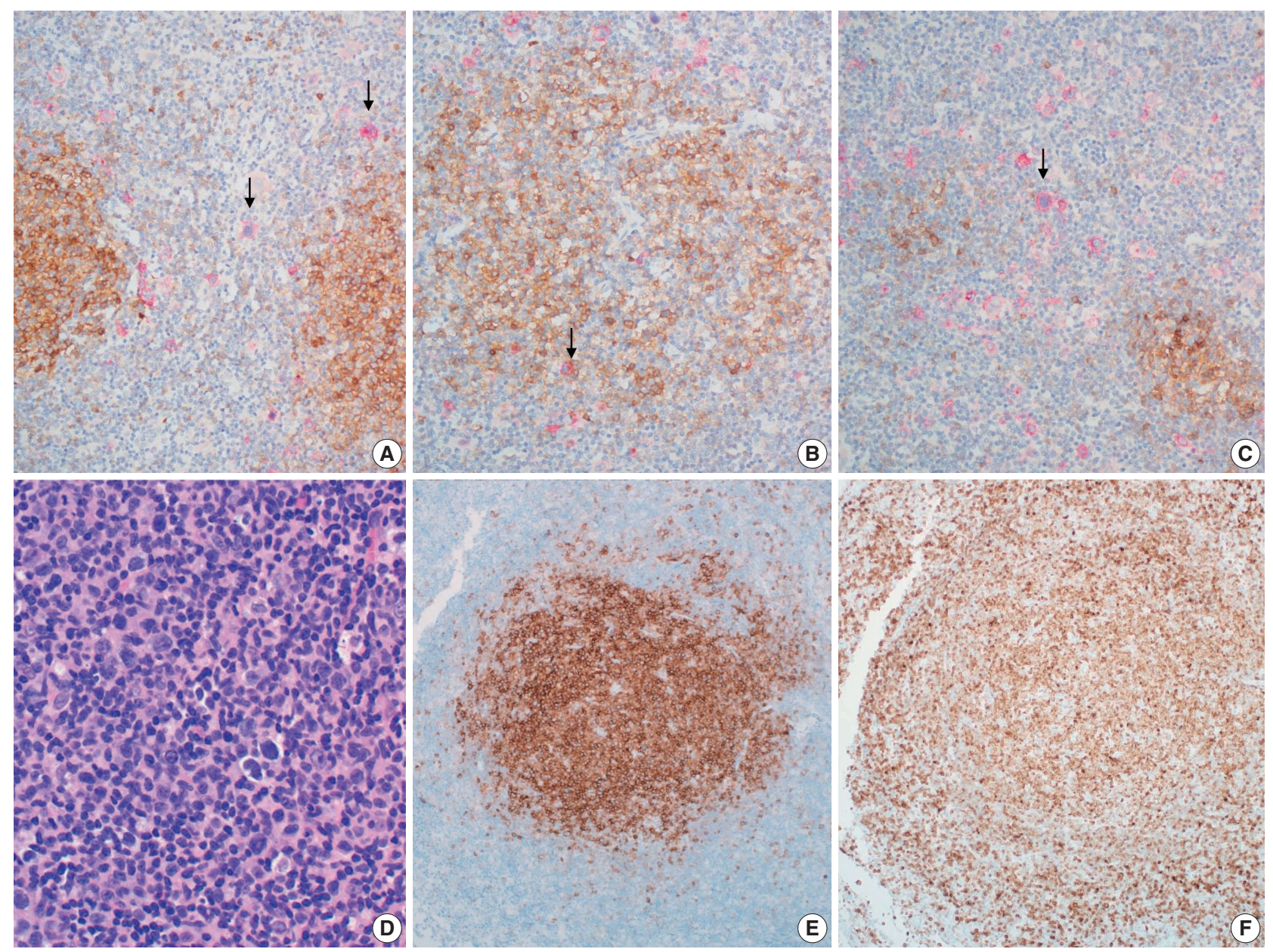

Fig. 2. (A-C) Composite follicular lymphoma and Hodgkin lymphoma of initial biopsy. Double stain for CD10 to show follicular lymphoma (brown) and CD30 to identify Hodgkin lymphoma (red). (A) CD30-positive Reed-Sternberg cells (arrows) are scattered in between follicular lymphoma. (B) A few CD30-positive Reed-Sternberg cells (arrow) within neoplastic follicles of follicular lymphoma. (C) Diffuse permeation of Hodgkin lymphoma to follicular lymphoma area. Reed-Sternberg cells (arrow) are scattered in diffuse area and small nodular remnant of neoplastic follicles. (D-F) Follicular lymphoma of recurrent lesion: (D) follicular lymphoma, grade 3a, (E) CD10, (F) BCL2.

In the initial biopsy of the present case, neoplastic follicles in the periphery of the lymph node were misinterpreted as a component of HL because the cellular atypia of the follicle was not recognized, and $\mathrm{HL}$ frequently has a vague follicular pattern.

While distinct clonal origin of separate tumor component of composite lymphoma has been reported in one study [12], it is well known that separate tumor components share a common clonal relationship, including a BCL2 translocation $[3,11,13]$. Recent next generation sequencing study revealed both components also share the same mutational variants but have pathogenic variants that are specific for each component as well [3]. These findings indicate that two distinct lymphomas sharing a common cytogenetic abnormality derived from the same precursor, overlaid with a subtype-specific mutation occurring in each subclone, can lead to composite HL and FL. In addition, EBV infection observed in HL cells, but not FL cells of the composite lymphoma [3] emphasizes the role of EBV infection in the pathogenesis of HL [17] and suggests that EBV infection also contributes to the formation of composite lymphoma. Composite lymphoma may pose both a diagnostic and managerial challenge [3]. A previous report demonstrated that the HL component in composite lymphoma pursues an indolent clinical course compared with de novo HL. No recurrence of the HL component was reported even without ABVD treatment for HL. In contrast, patients treated with ABVD only or who were untreated, experienced recurrence of FL like the present case [3]. Although the number of cases is too small to provide definitive information for treatment or to establish concrete guidelines for management [18], the above report may give a hint on how best to treat composite FL and HL. 


\section{Ethics Statement}

All procedures performed in the current study were approved by the Institutional Review Board (2021GR0492) in accordance with the 1964 Helsinki declaration and its later amendments. Informed consent was waived.

\section{Availability of Data and Material}

The datasets generated or analyzed during the study are available from the corresponding author on reasonable request.

\section{Code Availability}

Not applicable.

\section{ORCID}

Young Hyeh Ko https://orcid.org/0000-0002-4383-0579

Han-Na Kim https://orcid.org/0000-0001-5171-3658

Min Ji Jeon https://orcid.org/0000-0003-4044-5314

Eun Sang Yu https://orcid.org/0000-0003-2196-0732

Dae Sik Kim https://orcid.org/0000-0001-8424-8561

Chul-Won Choi https://orcid.org/0000-0002-3032-4239

\section{Author Contributions}

Conceptualization: MJJ, YHK. Review of data: HNK, ESY, DSK, CWC. Writing-original draft: HNK, YHK. Writing—review \& editing: MJJ, CWC, YHK.

\section{Conflicts of Interest}

The authors declare that they have no potential conflicts of interest.

\section{Funding Statement}

No funding to declare.

\section{References}

1. Custer R. Pitfalls in the diagnosis of lymphoma and leukemia from the pathologist's point of view. In: Proceedings of Second National Cancer Conference; 1952 Mar 3-5; Comcoinnati, OH, USA. New York: New York American Cancer Society, 1954; 554-7.

2. Kim H, Hendrickson R, Dorfman RF. Composite lymphoma. Cancer 1977; 40: 959-76.

3. Trecourt A, Mauduit C, Szablewski V, et al. Plasticity of mature B cells between follicular and classic Hodgkin lymphomas: a series of 22 cases expanding the spectrum of transdifferentiation. Am J Surg Pathol 2021 Jul 15 [Epub]. https://doi.org/10.1097/ PAS.0000000000001780.

4. Vasudevan JA, Nair RA, Sukumaran R, Nair SG. Composite lymphomas: Experience from a tertiary cancer center in Kerala, South India. Indian J Cancer 2017; 54: 358-61.

5. Tanaka J, Su P, Luedke C, et al. Composite lymphoma of follicular Bcell and peripheral T-cell types with distinct zone distribution in a 75-year-old male patient: a case study. Hum Pathol 2018; 76: 110-6.
6. Suefuji N, Niino D, Arakawa F, et al. Clinicopathological analysis of a composite lymphoma containing both T- and B-cell lymphomas. Pathol Int 2012; 62: 690-8.

7. Sun T, Susin M, Koduru P, et al. Phenotyping and genotyping of composite lymphoma with Ki-1 component. Hematol Pathol 1992; 6: 179-92.

8. York JC 2nd, Cousar JB, Glick AD, Flexner JM, Stein R, Collins RD. Morphologic and immunologic evidence of composite B- and Tcell lymphomas: a report of three cases developing in follicular center cell lymphomas. Am J Clin Pathol 1985; 84: 35-43.

9. Miyazawa Y, Yokohama A, Ishizaki T, et al. Pathological and molecular analysis of a composite lymphoma of mantle cell lymphoma and Epstein-Barr virus-positive follicular lymphoma. Int J Hematol 2021; 113: 592-9.

10. Almanaseer IY, Kosova L, Pellettiere EV. Composite lymphoma with immunoblastic features and Langerhans' cell granulomatosis (histiocytosis X). Am J Clin Pathol 1986; 85: 111-4.

11. Schmitz R, Renne C, Rosenquist R, et al. Insights into the multistep transformation process of lymphomas: IgH-associated translocations and tumor suppressor gene mutations in clonally related composite Hodgkin's and non-Hodgkin's lymphomas. Leukemia 2005; 19: $1452-8$.

12. Fend F, Quintanilla-Martinez L, Kumar S, et al. Composite low grade B-cell lymphomas with two immunophenotypically distinct cell populations are true biclonal lymphomas: a molecular analysis using laser capture microdissection. Am J Pathol 1999; 154: 185766.

13. Kuppers R, Sousa AB, Baur AS, Strickler JG, Rajewsky K, Hansmann ML. Common germinal-center B-cell origin of the malignant cells in two composite lymphomas, involving classical Hodgkin's disease and either follicular lymphoma or B-CLL. Mol Med 2001; 7: 285-92.

14. Thirumala S, Esposito M, Fuchs A. An unusual variant of composite lymphoma: a short case report and review of the literature. Arch Pathol Lab Med 2000; 124: 1376-8.

15. Brauninger A, Hansmann ML, Strickler JG, et al. Identification of common germinal-center B-cell precursors in two patients with both Hodgkin's disease and non-Hodgkin's lymphoma. N Engl J Med 1999; 340: 1239-47.

16. Gonzalez CL, Medeiros LJ, Jaffe ES. Composite lymphoma. A clinicopathologic analysis of nine patients with Hodgkin's disease and Bcell non-Hodgkin's lymphoma. Am J Clin Pathol 1991; 96: 81-9.

17. Mancao C, Altmann M, Jungnickel B, Hammerschmidt W. Rescue of "crippled" germinal 18.center B cells from apoptosis by EpsteinBarr virus. Blood 2005; 106: 4339-44.

18. Linck D, Lentini G, Tiemann M, Fauser AA, Parwaresch R, Basara $\mathrm{N}$. Sequential application of chemotherapy and monoclonal CD 20 antibody: successful treatment of advanced composite-lymphoma. Leuk Lymphoma 2005; 46: 285-8. 